\title{
A Note on the Partially Truncated Euler-Maruyama Method
}

\author{
Qian Guo ${ }^{1 *}$ Wei Liu ${ }^{1 \dagger}$, Xuerong $\mathrm{Mao}^{2 \ddagger}$ \\ ${ }^{1}$ Department of Mathematics, \\ Shanghai Normal University, Shanghai, China. \\ 2 Department of Mathematics and Statistics, \\ University of Strathclyde, Glasgow G1 1XH, U.K.
}

\begin{abstract}
The partially truncated Euler-Maruyama (EM) method was recently proposed in our earlier paper (Guo, Liu, Mao and Yue, 2017) for highly nonlinear stochastic differential equations (SDEs), where the finite-time strong $L^{r}$-convergence theory was established. In this note, we will point out that one condition imposed there is restrictive in the sense that this condition might force the stepsize to be so small that the partially truncated EM method would be inapplicable. In this note, we will remove this restrictive condition but still be able to establish the finite-time strong $L^{r}$-convergence rate. The advantages of our new results will be highlighted by the comparisons with our earlier results in (Guo, Liu, Mao and Yue, 2017).
\end{abstract}

Key words: Stochastic differential equation, local Lipschitz condition, Khasminskiitype condition, partially truncated Euler-Maruyama method, convergence rate.

\section{Introduction}

Influenced by Mao's papers [8, 9], we recently in [3] developed a new explicit numerical scheme, called the partially truncated EM method, for nonlinear SDEs under the local Lipschitz condi-

\footnotetext{
*E-mail:qguo@shnu.edu.cn

${ }^{\dagger}$ Corresponding author. E-mail:weiliu@shnu.edu.cn

†E-mail: x.mao@strath.ac.uk
} 
tion plus the Khasminskii-type condition. The real benefits of this new method lie in that the method can well preserve the asymptotic stability and boundedness of the underlying SDEs. However, we will point out in Section 2 that one condition imposed in [3] is restrictive in the sense that this condition might force the stepsize to be so small that the partially truncated EM method would be inapplicable. In this note, we will remove this restrictive condition but still be able to establish the finite-time strong $L^{r}$-convergence rate.

As this is the continuation of our earlier paper [3], we will use the same notation as used there. We will only list and cite some necessary references here but the reader can find more in $[3]$.

\section{Motivation}

In this section, we will point out that one condition imposed in [3] is restrictive in the sense that this condition might force the stepsize to be so small that the partially truncated EM method would be inapplicable. For this purpose, we need to make a quick review on the partially truncated EM method. Consider a $d$-dimensional SDE

$$
d x(t)=f(x(t)) d t+g(x(t)) d B(t)
$$

on $t \geq 0$ with the initial value $x(0)=x_{0} \in \mathbb{R}^{d}$, where $B(t)$ is an $m$-dimensional Brownian motion, $f: \mathbb{R}^{d} \rightarrow \mathbb{R}^{d}$ and $g: \mathbb{R}^{d} \rightarrow \mathbb{R}^{d \times m}$. We assume that $f$ and $g$ can be decomposed as

$$
f(x)=F_{1}(x)+F(x) \text { and } \quad g(x)=G_{1}(x)+G(x),
$$

where $F_{1}, F: \mathbb{R}^{d} \rightarrow \mathbb{R}^{d}$ and $G_{1}, G: \mathbb{R}^{d} \rightarrow \mathbb{R}^{d \times m}$ are all Borel measurable. The assumptions imposed in [3] are listed below.

Assumption 2.1 Assume that there are constants $L_{1}>0$ and $\gamma \geq 0$ such that

$$
\left|F_{1}(x)-F_{1}(y)\right| \vee\left|G_{1}(x)-G_{1}(y)\right| \leq L_{1}|x-y|
$$

and

$$
|F(x)-F(y)| \vee|G(x)-G(y)| \leq L_{1}\left(1+|x|^{\gamma}+|y|^{\gamma}\right)|x-y|
$$

for all $x, y \in \mathbb{R}^{d}$. 
Assumption 2.2 Assume that there is a pair of constants $\bar{r}>2$ and $L_{2}$ such that

$$
(x-y)^{T}(F(x)-F(y))+\frac{\bar{r}-1}{2}|G(x)-G(y)|^{2} \leq L_{2}|x-y|^{2}
$$

for all $x, y \in \mathbb{R}^{d}$.

Assumption 2.3 Assume that there are constants $\bar{p}>\bar{r}$ and $K_{2}>0$ such that

$$
x^{T} F(x)+\frac{\bar{p}-1}{2}|G(x)|^{2} \leq K_{2}\left(1+|x|^{2}\right)
$$

for all $x \in \mathbb{R}^{d}$.

As pointed out in [3], (2.6) cannot be deduced from (2.5) as we need $\bar{p}>\bar{r}$. Moreover, we observe from (2.3) that the coefficients $F_{1}$ and $G_{1}$ satisfy the linear growth condition, namely there exists a constant $K_{1}>0$ such that

$$
\left|F_{1}(x)\right| \vee\left|G_{1}(x)\right| \leq K_{1}(1+|x|)
$$

for all $x \in \mathbb{R}^{d}$.

To define the partially truncated EM numerical solutions, we first choose a strictly increasing continuous function $\mu: \mathbb{R}_{+} \rightarrow \mathbb{R}_{+}$such that $\mu(u) \rightarrow \infty$ as $u \rightarrow \infty$ and

$$
\sup _{|x| \leq u}(|F(x)| \vee|G(x)|) \leq \mu(u), \quad \forall u \geq 1
$$

Denote by $\mu^{-1}$ the inverse function of $\mu$ and we see that $\mu^{-1}$ is a strictly increasing continuous function from $[\mu(0), \infty)$ to $\mathbb{R}_{+}$. We also choose a strictly decreasing function $h:(0,1] \rightarrow(0, \infty)$ and a constant $\bar{h} \geq 1$ such that

$$
\lim _{\Delta \rightarrow 0} h(\Delta)=\infty \quad \text { and } \quad \Delta^{1 / 4} h(\Delta) \leq \bar{h}, \quad \forall \Delta \in(0,1]
$$

Before we proceed, let us make an useful remark.

Remark 2.4 In [3] where the partially truncated EM was originally developed, it was required to choose a number $\Delta^{*} \in(0,1]$ and a strictly decreasing function $h:\left(0, \Delta^{*}\right] \rightarrow(0, \infty)$ such that

$$
h\left(\Delta^{*}\right) \geq \mu(1), \quad \lim _{\Delta \rightarrow 0} h(\Delta)=\infty \quad \text { and } \quad \Delta^{1 / 4} h(\Delta) \leq 1, \quad \forall \Delta \in(0,1) .
$$

In this paper, we simply let $\Delta^{*}=1$ and remove the condition $h\left(\Delta^{*}\right) \geq \mu(1)$. In other words, we have made the choice of function $h$ much more flexible. We emphasise that these modifications 
do not make any effect on the results in [3]. In fact, the condition $h\left(\Delta^{*}\right) \geq \mu(1)$ was only used to show [3, Lemma 2.5] (followed from [8]). Instead of [3, Lemma 2.5], we will have our new Lemma 3.2. Comparing these two lemmas, we observe that the constant $2 K_{2}$ in [3, Lemma 2.5] is now replaced by $K_{4}$ in our our new Lemma 3.2. But this change does not have any effect on the results in [3]. We can also check that replacing $\Delta^{1 / 4} h(\Delta) \leq 1$ by $\Delta^{1 / 4} h(\Delta) \leq \bar{h}$ does not make any effect on the proof of [3, Lemma 3.2], which we will cite as Lemma 3.3 in this paper (in fact, such a change does not make any effect on any result in [3]).

For a given stepsize $\Delta \in(0,1]$, let us define the mapping $\pi_{\Delta}: \mathbb{R}^{d} \rightarrow \mathbb{R}^{d}$ by

$$
\pi_{\Delta}(x)=\left(|x| \wedge \mu^{-1}(h(\Delta))\right) \frac{x}{|x|}
$$

where we set $x /|x|=0$ when $x=0$. We then define the truncated functions

$$
F_{\Delta}(x)=F\left(\pi_{\Delta}(x)\right) \quad \text { and } \quad G_{\Delta}(x)=G\left(\pi_{\Delta}(x)\right)
$$

for $x \in \mathbb{R}^{d}$. It is easy to see that

$$
\left|F_{\Delta}(x)\right| \vee\left|G_{\Delta}(x)\right| \leq \mu\left(\mu^{-1}(h(\Delta))\right)=h(\Delta) \quad \forall x \in \mathbb{R}^{d}
$$

From now on we will define

$$
f_{\Delta}(x)=F_{1}(x)+F_{\Delta}(x) \quad \text { and } \quad g_{\Delta}(x)=G_{1}(x)+G_{\Delta}(x)
$$

The discrete-time partially truncated EM numerical solutions $X_{\Delta}\left(t_{k}\right) \approx x\left(t_{k}\right)$ for $t_{k}=k \Delta$ are formed by setting $X_{\Delta}(0)=x_{0}$ and computing

$$
X_{\Delta}\left(t_{k+1}\right)=X_{\Delta}\left(t_{k}\right)+f_{\Delta}\left(X_{\Delta}\left(t_{k}\right)\right) \Delta+g_{\Delta}\left(X_{\Delta}\left(t_{k}\right)\right) \Delta B_{k},
$$

for $k=0,1, \cdots$, where $\Delta B_{k}=B\left(t_{k+1}\right)-B\left(t_{k}\right)$. There are two versions of the continuous-time truncated EM solutions. The first one is defined by

$$
\bar{x}_{\Delta}(t)=\sum_{k=0}^{\infty} X_{\Delta}\left(t_{k}\right) I_{\left[t_{k}, t_{k+1}\right)}(t), \quad t \geq 0,
$$

where throughout this paper $I_{D}$ denotes the indicator function of set $D$. This is a simple step process so its sample paths are not continuous. We will refer to this as the continuous-time step-process partially truncated EM solution. The other one is defined by

$$
x_{\Delta}(t)=x_{0}+\int_{0}^{t} f_{\Delta}\left(\bar{x}_{\Delta}(s)\right) d s+\int_{0}^{t} g_{\Delta}\left(\bar{x}_{\Delta}(s)\right) d B(s)
$$


for $t \geq 0$. We will refer to this as the continuous-time continuous-sample partially truncated EM solution. We observe that $x_{\Delta}\left(t_{k}\right)=\bar{x}_{\Delta}\left(t_{k}\right)=X_{\Delta}\left(t_{k}\right)$ for all $k \geq 0$. Moreover, $x_{\Delta}(t)$ is an Itô process with its Itô differential

$$
d x_{\Delta}(t)=f_{\Delta}\left(\bar{x}_{\Delta}(t)\right) d t+g_{\Delta}\left(\bar{x}_{\Delta}(t)\right) d B(t) .
$$

The following theorem is one of the main results in [3].

Theorem 2.5 ([3, Theorem 3.1]) Let Assumptions 2.1, 2.2 and 2.3 hold and let $T>0$ be arbitrary. If $p \in(\bar{r}, \bar{p}), 2 p>\bar{r} \gamma$ and for $r \in[2, \bar{r})$

$$
h(\Delta) \geq \mu\left(\left(\Delta^{r / 2}(h(\Delta))^{r}\right)^{-1 /(p-r)}\right)
$$

then there is a $\bar{\Delta} \in\left(0, \Delta^{*}\right]$ such that for all $\Delta \in(0, \bar{\Delta}]$

$$
\mathbb{E}\left|x_{\Delta}(T)-x(T)\right|^{r} \leq C \Delta^{r / 2}(h(\Delta))^{r}
$$

and

$$
\mathbb{E}\left|\bar{x}_{\Delta}(T)-x(T)\right|^{r} \leq C \Delta^{r / 2}(h(\Delta))^{r},
$$

where throughout this paper $C$ stands for generic positive real constants.

Let us now explain, via an example, that condition (2.17) is somehow restrictive as it might force the stepsize to be so small that the partially truncated EM method would be inapplicable. Consider the scalar power logistic model in a population system (see, e.g., [1])

$$
d x(t)=x(t)\left[\left(5-10 x^{2}(t)\right) d t+x(t) d B(t)\right]
$$

where $B(t)$ is a scalar Brownian motion. Naturally, we can decompose the coefficients $f(x)=$ $5 x-10 x^{3}$ and $g(x)=x^{2}$ in the form of $(2.2)$ with

$$
F_{1}(x)=5 x, \quad F(x)=-10 x^{3}, \quad G_{1}(x)=0, \quad G(x)=x^{2} .
$$

Obviously, (2.3) is satisfied by $F_{1}$ and $G_{1}$, while it is easy to verify

$$
|F(x)-F(y)| \vee|G(x)-G(y)| \leq 15|x-y|\left(1+x^{2}+y^{2}\right), \quad \forall x, y \in \mathbb{R} .
$$

Thus, Assumption 2.1 is satisfied with $\gamma=2$. It is also not difficult to show that for $\bar{r}=3$,

$$
(x-y)(F(x)-F(y))+\frac{\bar{r}-1}{2}|G(x)-G(y)|^{2} \leq 0, \quad \forall x, y \in \mathbb{R},
$$


whence Assumption 2.2 is satisfied with $\bar{r}=3$. Moreover, for $\bar{p}=21$,

$$
x F(x)+\frac{\bar{p}-1}{2}|G(x)|^{2}=0, \quad \forall x, \in \mathbb{R},
$$

so Assumption 2.3 is satisfied with $\bar{p}=21$. Furthermore, we have

$$
\sup _{|x| \leq u}(|F(x)| \vee|G(x)|) \leq 10 u^{3}, \quad \forall u \geq 1
$$

whence we can choose $\mu(u)=10 u^{3}$. Finally choose $h(\Delta)=\Delta^{-1 / 6}$ which meets condition (2.9). Then, for $r=2$ and $p=20$, condition (2.17) becomes

$$
\Delta^{-1 / 6} \geq 10 \Delta^{-1 / 9}, \text { namely } \Delta \leq 10^{-18}
$$

This means we have to use an extremely small stepsize in order to apply Theorem 2.5 but this is not realistic. It is therefore very useful to remove this restrictive condition (2.17) and this is the main driven force for us to write this note.

\section{Main Results}

\subsection{Convergence rate}

It was shown in [3] that under Assumptions 2.1 and 2.3 , for any $p \in(2, \bar{p})$,

$$
x^{T} f(x)+\frac{p-1}{2}|g(x)|^{2} \leq K_{3}\left(1+|x|^{2}\right)
$$

for all $x \in \mathbb{R}^{d}$, where

$$
K_{3}=2 K_{1}+K_{2}+\frac{K_{1}^{2}(p-1)(\bar{p}-1)}{\bar{p}-p} .
$$

It was also shown in [3] that under Assumptions 2.1 and 2.2 , for any $q \in(2, \bar{r})$

$$
(x-y)^{T}(f(x)-f(y))+\frac{q-1}{2}|g(x)-g(y)|^{2} \leq L_{3}|x-y|^{2},
$$

where

$$
L_{3}=2 L_{1}+L_{2}+\frac{L_{1}^{2}(q-1)(\bar{r}-1)}{\bar{r}-q} .
$$

The following lemma is therefore well known (see, e.g., $[5,7,11]$ ).

Lemma 3.1 Under Assumptions 2.1 and 2.3, the SDE (2.1) has a unique global solution $x(t)$ and, moreover, for any $p \in(2, \bar{p})$,

$$
\sup _{0 \leq t \leq T} \mathbb{E}|x(t)|^{p}<\infty, \quad \forall T>0 .
$$


The following lemma shows that the truncated functions $F_{\Delta}$ and $G_{\Delta}$ preserve Assumption 2.3 very well.

Lemma 3.2 Let Assumption 2.3 hold. Then, for all $\Delta \in(0,1]$, we have

$$
x^{T} F_{\Delta}(x)+\frac{\bar{p}-1}{2}\left|G_{\Delta}(x)\right|^{2} \leq K_{4}\left(1+|x|^{2}\right), \quad \forall x \in \mathbb{R}^{d},
$$

where $K_{4}=2 K_{2}\left(1 \vee\left[1 / \mu^{-1}(h(1))\right]\right)$.

Proof. This lemma was essentially proved in $[3,8]$ but we here do not need condition $h\left(\Delta^{*}\right) \geq$ $\mu(1)$ as we pointed out in Remark 2.4.

Fix any $\Delta \in(0,1]$. For $x \in \mathbb{R}^{d}$ with $|x| \leq \mu^{-1}(h(\Delta))$, the required assertion (3.4) holds clearly. For $x \in \mathbb{R}^{d}$ with $|x|>\mu^{-1}(h(\Delta))$, the proof of [8, Lemma 2.4] shows that

$$
x^{T} F_{\Delta}(x)+\frac{\bar{p}-1}{2}\left|G_{\Delta}(x)\right|^{2} \leq \frac{|x|}{\mu^{-1}(h(\Delta))} K_{2}\left(1+\left[\mu^{-1}(h(\Delta))\right]^{2}\right) .
$$

Noting that $\mu^{-1}(h(\Delta)) \geq \mu^{-1}(h(1))$, we then derive

$$
\begin{aligned}
& x^{T} F_{\Delta}(x)+\frac{\bar{p}-1}{2}\left|G_{\Delta}(x)\right|^{2} \leq K_{2}|x|\left(\frac{1}{\mu^{-1}(h(1))}+|x|\right) \\
\leq & K_{2}\left(1 \vee\left[1 / \mu^{-1}(h(1))\right]\right)\left(|x|+|x|^{2}\right) \leq 0.5 K_{4}\left(1+2|x|^{2}\right) \leq K_{4}\left(1+|x|^{2}\right)
\end{aligned}
$$

as required.

As we explained in Remark 2.4, this lemma plays the same role as [3, Lemma 2.5] did and all the results in [3] work although our new condition $(2.9)$ on the function $h(\cdot)$ is simpler. In particular, we can then cite a lemma from [3] for the use of this paper.

Lemma 3.3 Let Assumptions 2.1 and 2.3 hold. Let $p \in(2, \bar{p})$ and $T>0$ be arbitrary. Then

$$
\sup _{0<\Delta \leq 1} \sup _{0 \leq t \leq T} \mathbb{E}\left|x_{\Delta}(t)\right|^{p} \leq C
$$

(We emphasise once again that $C$ stands for generic positive real constants dependent on $T, \bar{p}, p, x_{0}, K_{1}, K_{2}, \bar{h}$ etc. but independent of $\Delta$ and its values may change between occurrences.)

The following lemma was essentially proved in [3].

Lemma 3.4 Let Assumptions 2.1 and 2.3 hold. Let $p \in(0, \bar{p})$ and $T>0$ be arbitrary. Then, for every $\Delta \in(0,1]$,

$$
\mathbb{E}\left|x_{\Delta}(t)-\bar{x}_{\Delta}(t)\right|^{p} \leq C \Delta^{p / 2}(h(\Delta))^{p}, \quad \forall t \in[0, T]
$$


Proof. This lemma was proved in [3] for $p \in(2, \bar{p})$ in the sense that there is a $\bar{\Delta} \in(0,1]$ such that assertion $(3.6)$ holds for all $\Delta \in(0, \bar{\Delta}]$. Our new version here is therefore an improvement of that in [3]. It is sufficient to show our lemma for $p \in(2, \bar{p})$ as the assertion for $p \in(0,2]$ follows then from the Hölder inequality. We hence fix $p \in(2, \bar{p})$ arbitrarily. It follows from Lemma 3.3 that

$$
\sup _{0<\Delta \leq 1} \sup _{0 \leq t \leq T} \mathbb{E}\left|x_{\Delta}(t)\right|^{p} \leq C(h(1))^{p} .
$$

Now, fix any $\Delta \in(0,1]$. For any $t \in[0, T]$, there is a unique $k \geq 0$ such that $t_{k} \leq t \leq t_{k+1}$. By (2.7) and (2.11), it is easy to show from (2.15) that

$$
\mathbb{E}\left|x_{\Delta}(t)-\bar{x}_{\Delta}(t)\right|^{p} \leq C \Delta^{p / 2}\left(1+\mathbb{E}\left|\bar{x}_{\Delta}(t)\right|^{p}+(h(\Delta))^{p}\right)
$$

But, by (3.7) and the decreasing property of $h$,

$$
\mathbb{E}\left|\bar{x}_{\Delta}(t)\right|^{p} \leq C(h(1))^{p} \leq C(h(\Delta))^{p}
$$

We hence get assertion (3.6) from (3.8).

Let us now present one more lemma which will be used in the proof of our main theorem later.

Lemma 3.5 Let Assumption 2.1 hold. Then

$$
|f(x)-f(y)| \vee|g(x)-g(y)| \leq 2 L_{1}\left(1+|x|^{\gamma}+|y|^{\gamma}\right)|x-y|
$$

for all $x, y \in \mathbb{R}^{d}$. Moreover, for any $\Delta \in(0,1]$,

$$
\left|f_{\Delta}(x)-f_{\Delta}(y)\right| \vee\left|g_{\Delta}(x)-g_{\Delta}(y)\right| \leq 2 L_{1}\left(1+|x|^{\gamma}+|y|^{\gamma}\right)|x-y|
$$

for all $x, y \in \mathbb{R}^{d}$.

Proof. Assertion (3.9) follows easily from Assumption 2.1. To show assertion (3.10), we first show that the truncated functions $F_{\Delta}$ and $G_{\Delta}$ preserve condition (2.4) perfectly. In fact, by condition (2.4), we have

$$
\begin{aligned}
& \left|F_{\Delta}(x)-F_{\Delta}(y)\right| \vee\left|G_{\Delta}(x)-G_{\Delta}(y)\right| \\
= & \left|F\left(\pi_{\Delta}(x)\right)-F\left(\pi_{\Delta}(y)\right)\right| \vee\left|G\left(\pi_{\Delta}(x)\right)-G\left(\pi_{\Delta}(y)\right)\right| \\
\leq & L_{1}\left(1+\left|\pi_{\Delta}(x)\right|^{\gamma}+\left|\pi_{\Delta}(y)\right|^{\gamma}\right)\left|\pi_{\Delta}(x)-\pi_{\Delta}(y)\right|
\end{aligned}
$$


for all $x, y \in \mathbb{R}^{d}$. Noting

$$
\left|\pi_{\Delta}(x)\right| \leq|x|, \quad\left|\pi_{\Delta}(y)\right| \leq|y|, \quad\left|\pi_{\Delta}(x)-\pi_{\Delta}(y)\right| \leq|x-y|
$$

we get

$$
\left|F_{\Delta}(x)-F_{\Delta}(y)\right| \vee\left|G_{\Delta}(x)-G_{\Delta}(y)\right| \leq L_{1}\left(1+|x|^{\gamma}+|y|^{\gamma}\right)|x-y| .
$$

This, together with condition (2.3), implies the other assertion (3.10).

Moreover, we also observe from Assumption 2.1 that

$$
|F(x)| \vee|G(x)| \leq L_{4}|x|^{(1+\gamma)}, \quad \forall|x| \geq 1
$$

where $L_{4}=2 L_{1}+|F(0)|+|G(0)|$.

The following theorem is our main result in this paper which shows the rate of $L^{r}$ convergence. From now on, we will fix $T>0$ arbitrarily.

Theorem 3.6 Let Assumptions 2.1, 2.2, 2.3 hold and assume that there is a number $p \in(2, \bar{p})$ such that

$$
p>(1+\gamma) \bar{r}
$$

Let $r \in[2, \bar{r})$ be arbitrary. Then for any $\Delta \in(0,1]$,

$$
\mathbb{E}\left|x(T)-x_{\Delta}(T)\right|^{r} \leq C\left(\left(\mu^{-1}(h(\Delta))\right)^{-(p-(1+\gamma) r)}+\Delta^{r / 2}(h(\Delta))^{r}\right)
$$

and

$$
\mathbb{E}\left|x(T)-\bar{x}_{\Delta}(T)\right|^{r} \leq C\left(\left(\mu^{-1}(h(\Delta))\right)^{-(p-(1+\gamma) r)}+\Delta^{r / 2}(h(\Delta))^{r}\right) .
$$

In particular, recalling (3.12), we may define

$$
\mu(u)=L_{4} u^{(1+\gamma)}, \quad u \geq 0,
$$

and let

$$
h(\Delta)=\bar{h} \Delta^{-\varepsilon} \quad \text { for some } \varepsilon \in(0,1 / 4]
$$

to get

$$
\mathbb{E}\left|x(T)-x_{\Delta}(T)\right|^{r} \leq C \Delta^{[\varepsilon(p-(1+\gamma) r) /(1+\gamma)] \wedge[r(1-2 \varepsilon) / 2]}
$$

and

$$
\mathbb{E}\left|x(T)-\bar{x}_{\Delta}(T)\right|^{r} \leq C \Delta^{[\varepsilon(p-(1+\gamma) r) /(1+\gamma)] \wedge[r(1-2 \varepsilon) / 2]}
$$

for all $\Delta \in(0,1]$. 
Proof. First of all, fix a number $q \in(r, \bar{r})$. By (3.13), we have

$$
p>(1+\gamma) q .
$$

We also fix $\Delta \in(0,1]$ arbitrarily. Let $\zeta_{\Delta}(t)=x(t)-x_{\Delta}(t)$ for $t \geq 0$. For each integer $n>\left|x_{0}\right|$, define the stopping time

$$
\rho_{n}=\inf \left\{t \geq 0:|x(t)| \vee\left|x_{\Delta}(t)\right| \geq n\right\},
$$

where we set $\inf \emptyset=\infty$ (as usual $\emptyset$ denotes the empty set). By the Itô formula, we have that for $0 \leq t \leq T$,

$$
\begin{gathered}
\mathbb{E}\left|\zeta_{\Delta}\left(t \wedge \rho_{n}\right)\right|^{r} \leq \mathbb{E} \int_{0}^{t \wedge \rho_{n}} r\left|\zeta_{\Delta}(s)\right|^{r-2}\left(\zeta_{\Delta}^{T}(s)\left[f(x(s))-f_{\Delta}\left(\bar{x}_{\Delta}(s)\right)\right]\right. \\
\left.+\frac{r-1}{2}\left|g(x(s))-g_{\Delta}\left(\bar{x}_{\Delta}(s)\right)\right|^{2}\right) d s
\end{gathered}
$$

Noting

$$
\begin{aligned}
& \frac{r-1}{2}\left|g(x(s))-g_{\Delta}\left(\bar{x}_{\Delta}(s)\right)\right|^{2} \\
\leq & \frac{r-1}{2}\left[\left(1+\frac{q-r}{r-1}\right)\left|g(x(s))-g\left(x_{\Delta}(s)\right)\right|^{2}+\left(1+\frac{r-1}{q-r}\right)\left|g\left(x_{\Delta}(s)\right)-g_{\Delta}\left(\bar{x}_{\Delta}(s)\right)\right|^{2}\right] \\
= & \frac{q-1}{2}\left|g(x(s))-g\left(x_{\Delta}(s)\right)\right|^{2}+\frac{(r-1)(q-1)}{2(q-r)}\left|g\left(x_{\Delta}(s)\right)-g\left(\bar{x}_{\Delta}(s)\right)\right|^{2},
\end{aligned}
$$

we get from (3.21) that

$$
\mathbb{E}\left|\zeta_{\Delta}\left(t \wedge \rho_{n}\right)\right|^{r} \leq H_{1}+H_{2},
$$

where

$$
\begin{aligned}
H_{1}=\mathbb{E} \int_{0}^{t \wedge \rho_{n}} r\left|\zeta_{\Delta}(s)\right|^{r-2}( & \zeta_{\Delta}^{T}(s)\left[f(x(s))-f\left(x_{\Delta}(s)\right)\right] \\
& \left.+\frac{q-1}{2}\left|g(x(s))-g\left(x_{\Delta}(s)\right)\right|^{2}\right) d s
\end{aligned}
$$

and

$$
\begin{aligned}
H_{2}=\mathbb{E} \int_{0}^{t \wedge \rho_{n}} r\left|\zeta_{\Delta}(s)\right|^{r-2}\left(\zeta_{\Delta}^{T}(s)\left[f\left(x_{\Delta}(s)\right)-f_{\Delta}\left(\bar{x}_{\Delta}(s)\right)\right]\right. \\
\left.\quad+\frac{(r-1)(q-1)}{2(q-r)}\left|g\left(x_{\Delta}(s)\right)-g_{\Delta}\left(\bar{x}_{\Delta}(s)\right)\right|^{2}\right) d s
\end{aligned}
$$

By (3.2), we have

$$
H_{1} \leq r L_{3} \mathbb{E} \int_{0}^{t \wedge \rho_{n}}\left|\zeta_{\Delta}(s)\right|^{r} d s .
$$


It is also easy to see that

$$
H_{2} \leq H_{3}+H_{4},
$$

where

$$
\begin{array}{rl}
H_{3}=\mathbb{E} \int_{0}^{t \wedge \rho_{n}} r & r\left|\zeta_{\Delta}(s)\right|^{r-2}\left(\zeta_{\Delta}^{T}(s)\left[f\left(x_{\Delta}(s)\right)-f_{\Delta}\left(x_{\Delta}(s)\right)\right]\right. \\
& \left.+\frac{(r-1)(q-1)}{(q-r)}\left|g\left(x_{\Delta}(s)\right)-g_{\Delta}\left(x_{\Delta}(s)\right)\right|^{2}\right) d s
\end{array}
$$

and

$$
\begin{aligned}
H_{4}=\mathbb{E} \int_{0}^{t \wedge \rho_{n}} r\left|\zeta_{\Delta}(s)\right|^{r-2}\left(\zeta_{\Delta}^{T}(s)\left[f_{\Delta}\left(x_{\Delta}(s)\right)-f_{\Delta}\left(\bar{x}_{\Delta}(s)\right)\right]\right. \\
\left.\quad+\frac{(r-1)(q-1)}{(q-r)}\left|g_{\Delta}\left(x_{\Delta}(s)\right)-g_{\Delta}\left(\bar{x}_{\Delta}(s)\right)\right|^{2}\right) d s .
\end{aligned}
$$

Let us estimate $H_{4}$ first. By (3.10), we have

$$
\begin{aligned}
H_{4} \leq & \mathbb{E} \int_{0}^{t \wedge \rho_{n}} r\left|\zeta_{\Delta}(s)\right|^{r-2}\left(0.5\left|\zeta_{\Delta}(s)\right|^{2}+0.5\left|f_{\Delta}\left(x_{\Delta}(s)\right)-f_{\Delta}\left(\bar{x}_{\Delta}(s)\right)\right|^{2}\right. \\
& \left.\quad+\frac{(r-1)(q-1)}{(q-r)}\left|g_{\Delta}\left(x_{\Delta}(s)\right)-g_{\Delta}\left(\bar{x}_{\Delta}(s)\right)\right|^{2}\right) d s \\
& \leq 0.5 r \mathbb{E} \int_{0}^{t \wedge \rho_{n}}\left|\zeta_{\Delta}(s)\right|^{r} d s \\
& +C \mathbb{E} \int_{0}^{t \wedge \rho_{n}}\left|\zeta_{\Delta}(s)\right|^{r-2}\left(1+\left|x_{\Delta}(s)\right|^{2 \gamma}+\left|\bar{x}_{\Delta}(s)\right|^{2 \gamma}\right)\left|x_{\Delta}(s)-\bar{x}_{\Delta}(s)\right|^{2} d s .
\end{aligned}
$$

But, by the well-known Young inequality (see, e.g., [10, p.52]),

$$
\begin{aligned}
& \left|\zeta_{\Delta}(s)\right|^{r-2}\left(1+\left|x_{\Delta}(s)\right|^{2 \gamma}+\left|\bar{x}_{\Delta}(s)\right|^{2 \gamma}\right)\left|x_{\Delta}(s)-\bar{x}_{\Delta}(s)\right|^{2} \\
\leq & \frac{r-2}{r}\left|\zeta_{\Delta}(s)\right|^{r}+\frac{2}{r}\left(1+\left|x_{\Delta}(s)\right|^{2 \gamma}+\left|\bar{x}_{\Delta}(s)\right|^{2 \gamma}\right)^{r / 2}\left|x_{\Delta}(s)-\bar{x}_{\Delta}(s)\right|^{r} \\
\leq & \left|\zeta_{\Delta}(s)\right|^{r}+C\left(1+\left|x_{\Delta}(s)\right|^{\gamma r}+\left|\bar{x}_{\Delta}(s)\right|^{\gamma r}\right)\left|x_{\Delta}(s)-\bar{x}_{\Delta}(s)\right|^{r} .
\end{aligned}
$$

Substituting this into (3.27) yields

$$
\begin{aligned}
H_{4} & \leq C \mathbb{E} \int_{0}^{t \wedge \rho_{n}}\left|\zeta_{\Delta}(s)\right|^{r} d s \\
& +C \int_{0}^{T} \mathbb{E}\left(\left(1+\left|x_{\Delta}(s)\right|^{\gamma r}+\left|\bar{x}_{\Delta}(s)\right|^{\gamma r}\right)\left|x_{\Delta}(s)-\bar{x}_{\Delta}(s)\right|^{r}\right) d s .
\end{aligned}
$$

By the Hölder inequality,

$$
\begin{aligned}
& \mathbb{E}\left(\left(1+\left|x_{\Delta}(s)\right|^{\gamma r}+\left|\bar{x}_{\Delta}(s)\right|^{\gamma r}\right)\left|x_{\Delta}(s)-\bar{x}_{\Delta}(s)\right|^{r}\right) \\
\leq & 3^{\gamma r(p-1) / p}\left(\mathbb{E}\left(1+\left|x_{\Delta}(s)\right|^{p}+\left|\bar{x}_{\Delta}(s)\right|^{p}\right)\right)^{\gamma r / p}\left(\mathbb{E}\left|x_{\Delta}(s)-\bar{x}_{\Delta}(s)\right|^{p r /(p-\gamma r)}\right)^{(p-\gamma r) / p} .
\end{aligned}
$$


But, by (3.20), we have $p>(1+\gamma) r$ and hence $p r /(p-\gamma r)<p$. So we can apply Lemmas 3.3 and 3.4 to get

$$
\mathbb{E}\left(\left(1+\left|x_{\Delta}(s)\right|^{\gamma r}+\left|\bar{x}_{\Delta}(s)\right|^{\gamma r}\right)\left|x_{\Delta}(s)-\bar{x}_{\Delta}(s)\right|^{r}\right) \leq C \Delta^{r / 2}(h(\Delta))^{r}
$$

Substituting this into (3.28) gives

$$
H_{4} \leq \mathbb{E} \int_{0}^{t \wedge \rho_{n}}\left|\zeta_{\Delta}(s)\right|^{r} d s+C \Delta^{r / 2}(h(\Delta))^{r}
$$

Let us now estimate $H_{3}$. It is easy to show that

$$
H_{3} \leq C \mathbb{E} \int_{0}^{t \wedge \rho_{n}}\left|\zeta_{\Delta}(s)\right|^{r} d s+H_{5}
$$

where

$$
H_{5}=C \int_{0}^{T}\left(\mathbb{E}\left|f\left(x_{\Delta}(s)\right)-f_{\Delta}\left(x_{\Delta}(s)\right)\right|^{r}+\mathbb{E}\left|g\left(x_{\Delta}(s)\right)-g_{\Delta}\left(x_{\Delta}(s)\right)\right|^{r}\right) d s .
$$

Recalling (2.2), (2.12) and (2.10), we see

$$
\mathbb{E}\left|f\left(x_{\Delta}(s)\right)-f_{\Delta}\left(x_{\Delta}(s)\right)\right|^{r}=\mathbb{E}\left|F\left(x_{\Delta}(s)\right)-F_{\Delta}\left(x_{\Delta}(s)\right)\right|^{r}=\mathbb{E}\left|F\left(x_{\Delta}(s)\right)-F\left(\pi_{\Delta}\left(x_{\Delta}(s)\right)\right)\right|^{r} .
$$

By (2.4), the Hölder inequality and Lemma 3.3, we then derive

$$
\begin{aligned}
& \mathbb{E}\left|f\left(x_{\Delta}(s)\right)-f_{\Delta}\left(x_{\Delta}(s)\right)\right|^{r} \\
\leq & C \mathbb{E}\left(\left(1+\left|x_{\Delta}(s)\right|^{\gamma r}+\left|\pi_{\Delta}\left(x_{\Delta}(s)\right)\right|^{\gamma r}\right)\left|x_{\Delta}(s)-\pi_{\Delta}\left(x_{\Delta}(s)\right)\right|^{r}\right) \\
\leq & C\left(\mathbb{E}\left(1+\left|x_{\Delta}(s)\right|^{p}+\left|\pi_{\Delta}\left(x_{\Delta}(s)\right)\right|^{p}\right)\right)^{\gamma r / p}\left(\mathbb{E}\left|x_{\Delta}(s)-\pi_{\Delta}\left(x_{\Delta}(s)\right)\right|^{p r /(p-\gamma r)}\right)^{(p-\gamma r) / p} \\
\leq & C\left(\mathbb{E}\left[I_{\left.\left.\left\{\left|x_{\Delta}(s)\right|>\mu^{-1}(h(\Delta))\right\}\left|x_{\Delta}(s)\right|^{p r /(p-\gamma r)}\right]\right)^{(p-\gamma r) / p}}\left[\mathbb{E}\left|x_{\Delta}(s)\right|^{p}\right]^{r /(p-\gamma r)}\right)^{(p-\gamma r) / p}\right. \\
\leq & C\left(\left[\mathbb{P}\left\{\left|x_{\Delta}(s)\right|>\mu^{-1}(h(\Delta))\right\}\right]^{(p-(1+\gamma) r) /(p-\gamma r)}\left[\begin{array}{l}
\mathbb{E}\left|x_{\Delta}(s)\right|^{p} \\
\leq
\end{array}\right)^{(p-(1+\gamma) r) / p}\left(\mu^{-1}(h(\Delta))\right)^{p}\right. \\
\leq & C\left(\mu^{-1}(h(\Delta))\right)^{-(p-(1+\gamma) r)} .
\end{aligned}
$$

Similarly, we can show

$$
\mathbb{E}\left|g\left(x_{\Delta}(s)\right)-g_{\Delta}\left(x_{\Delta}(s)\right)\right|^{r} \leq C\left(\mu^{-1}(h(\Delta))\right)^{-(p-(1+\gamma) r)} .
$$

Substituting the two inequalities into (3.31) and then putting the resulting inequality into (3.30), we obtain

$$
H_{3} \leq C \mathbb{E} \int_{0}^{t \wedge \rho_{n}}\left|\zeta_{\Delta}(s)\right|^{r} d s+C\left(\mu^{-1}(h(\Delta))\right)^{-(p-(1+\gamma) r)}
$$


Combining (3.22), (3.25), (3.26, (3.29) and (3.32) together we get

$$
\begin{aligned}
& \mathbb{E}\left|\zeta_{\Delta}\left(t \wedge \rho_{n}\right)\right|^{r} \\
\leq & C\left(\mathbb{E} \int_{0}^{t \wedge \rho_{n}}\left|\zeta_{\Delta}(s)\right|^{r} d s+\left(\mu^{-1}(h(\Delta))\right)^{-(p-(1+\gamma) r)}+\Delta^{r / 2}(h(\Delta))^{r}\right) \\
\leq & C\left(\int_{0}^{t} \mathbb{E}\left|\zeta_{\Delta}\left(s \wedge \rho_{n}\right)\right|^{r} d s+\left(\mu^{-1}(h(\Delta))\right)^{-(p-(1+\gamma) r)}+\Delta^{r / 2}(h(\Delta))^{r}\right) .
\end{aligned}
$$

An application of the Gronwall inequality yields that

$$
\mathbb{E}\left|\zeta_{\Delta}\left(T \wedge \rho_{n}\right)\right|^{r} \leq C\left(\left(\mu^{-1}(h(\Delta))\right)^{-(p-(1+\gamma) r)}+\Delta^{r / 2}(h(\Delta))^{r}\right)
$$

This implies the desired assertion (3.14) by letting $n \rightarrow \infty$. The another assertion (3.15) follows from (3.14) and Lemma 3.4.

Finally, when $\mu$ is defined by (3.16), then $\mu^{-1}(u)=\left(u / L_{4}\right)^{1 /(1+\gamma)}$. Substituting this and (3.17) into (3.14) we get

$$
\mathbb{E}|x(T)-x \Delta(T)|^{r} \leq C\left(\Delta^{\varepsilon(p-(1+\gamma) r) /(1+\gamma)}+\Delta^{r(1-2 \varepsilon) / 2}\right),
$$

which is the required assertion (3.18). Similarly, we can show (3.19). The proof is therefore complete.

The following corollary shows that the order of $L^{r}$-convergence is close to $1 / 2$. This is almost optimal if we recall that the classical EM method has order $1 / 2$ of $L^{r}$-convergence under the global Lipschitz condition.

Corollary 3.7 Let Assumptions 2.1, 2.2 hold and let Assumption 2.3 hold for any $\bar{p}>\bar{r}$. Let $\mu(\cdot)$ and $h(\cdot)$ be defined by (3.16) and (3.17). Then, for any $r \in[2, \bar{r})$ and any $\varepsilon \in(0,1 / 4)$,

$$
\mathbb{E}\left|x(T)-x_{\Delta}(T)\right|^{r} \leq C \Delta^{r(1-2 \varepsilon) / 2}
$$

and

$$
\mathbb{E}\left|x(T)-\bar{x}_{\Delta}(T)\right|^{r} \leq C \Delta^{r(1-2 \varepsilon) / 2} .
$$

Proof. Choosing $p$ sufficiently large for

$$
\varepsilon(p-(1+\gamma) r) /(1+\gamma)>r(1-2 \varepsilon) / 2,
$$

we get the assertions from (3.18) and (3.19).

Let us discuss an example to illustrate this corollary before we make a comparison between our new Theorem 3.6 and our earlier result, namely Theorem 2.5. 
Example 3.8 Consider the scalar stochastic Ginzburgh-Landau equation (see, e.g., [2, 6])

$$
d x(t)=\left(a x(t)-b x^{3}(t)\right) d t+c x(t) d B(t)
$$

where $B(t)$ is a scalar Brownian motion and $a, b, c$ are three positive numbers. We naturally decompose the coefficients $f(x)=a x-b x^{3}$ and $g(x)=c x$ in the form of (2.2) with

$$
F_{1}(x)=a x, \quad F(x)=b x^{3}, \quad G_{1}(x)=c x, \quad G(x)=0
$$

for $x \in \mathbb{R}$. Both $F_{1}(x)$ and $G_{1}(x)$ are linear functions of $x$ so they satisfy condition (2.3) of course. Also, for $x, y \in \mathbb{R}$,

$$
|F(x)-F(y)|=b\left(x^{2}+x y+y^{2}\right)|x-y| \leq 2 b\left(x^{2}+y^{2}\right)|x-y| .
$$

Thus, Assumption 2.1 is satisfied with $\gamma=2$ as $G(x)=0$. Moreover, for any $\bar{r}>2$

$$
(x-y)(F(x)-F(y))+\frac{\bar{r}-1}{2}|G(x)-G(y)|^{2}=-b\left(x^{2}+x y+y^{2}\right)(x-y)^{2} \leq 0 .
$$

This means that Assumption 2.2 holds for any $\bar{r}>2$. Furthermore, for any $\bar{p}>2$,

$$
x F(x)+\frac{\bar{p}-1}{2}|G(x)|^{2}=-b x^{4} \leq 0 .
$$

Hence, Assumption 2.3 is also satisfied for any $\bar{p}>2$. To apply Corollary 3.7, we still need to design functions $\mu$ and $h$. Noting that

$$
\sup _{|x| \leq u}(|F(x)| \vee|G(x)|) \leq(b \vee c) u^{3}, \quad \forall u \geq 1,
$$

we can have $\mu(u)=(b \vee v) u^{3}$ and its inverse function $\mu^{-1}(u)=(u /(b \vee c))^{1 / 3}$ for $u \geq 0$. For $\varepsilon \in(0,1 / 4]$, we define $h(\Delta)=\bar{h} \Delta^{-\varepsilon}$ for $\Delta>0$. Obviously, (2.9) holds. We can therefore conclude by Corollary 3.7 that for any $r \geq 2$, the partially truncated EM solutions of the SDE (3.36) satisfy

$$
\mathbb{E}\left|x(T)-x_{\Delta}(T)\right|^{r} \leq C \Delta^{r(1-2 \varepsilon) / 2} \quad \text { and } \quad \mathbb{E}\left|x(T)-\bar{x}_{\Delta}(T)\right|^{r} \leq C\left(\Delta^{r(1-2 \varepsilon) / 2}\right) .
$$

That is, the order of $L^{r}$-convergence can be arbitrarily close to $1 / 2$.

\subsection{Comparison}

It is time to compare our new Theorem 3.6 with our earlier result, namely Theorem 2.5. Although both theorems are formed under the same set of assumptions (i.e., Assumptions 2.1, 2.2 and 2.3), we observe the following key differences: 
- Theorem 3.6 does not need condition (2.17) in Theorem 2.5.

- The assertions of Theorem 3.6 hold for any $\Delta \in(0,1]$ while the assertions of Theorem 2.5 holds only for sufficiently small $\Delta$ which satisfies condition (2.17).

- Condition $p>(1+\gamma) \bar{r}$ in Theorem 3.6 is stronger than $2 p>\gamma \bar{r}$ in Theorem 2.5.

- The convergence order showed by Theorem 3.6 is worse than that by Theorem 2.5, although could be the same when $p$ is sufficiently large as Corollary 3.7 has shown.

The key advantage of our new Theorem 3.6 lies in that it does not need condition (2.17). We have already seen in Section 2 that condition (2.17) could sometimes make Theorem 2.5 inapplicable.

Example 3.9 Let us return to the SDE (2.20) and show that our new Theorem 3.6 is indeed applicable. We have already verified that Assumptions 2.1, 2.2 and 2.3 hold with $\gamma=2, \bar{r}=3$ and $\bar{p}=21$. We also have $\mu(u)=3 u^{3}$.

Let us first let $h(\Delta)=\Delta^{-1 / 5}$. For $r=2$ and $p=20$, condition (2.17) becomes

$$
\Delta^{-1 / 5} \geq 10^{-1 / 10}, \text { namely } \Delta \leq 10^{-10}
$$

and Theorem 2.5 shows that the partially truncated EM method applied to the SDE (2.20) has the property that

$$
\mathbb{E}\left|x(T)-x_{\Delta}(T)\right|^{2} \vee \mathbb{E}\left|x(T)-\bar{x}_{\Delta}(T)\right|^{2} \leq C \Delta^{3 / 5}
$$

On the other hand, all the conditions of our new Theorem 3.6 are satisfied and hence we can conclude that

$$
\mathbb{E}\left|x(T)-x_{\Delta}(T)\right|^{2} \vee \mathbb{E}\left|x(T)-\bar{x}_{\Delta}(T)\right|^{2} \leq C\left(\Delta^{14 / 15}+\Delta^{3 / 5}\right) \leq C \Delta^{3 / 5}
$$

for any stepsize $\Delta \in(0,1]$. This shows the same convergence rate as (3.37) did but we have no longer required $\Delta \leq 10^{-10}$.

To improve the convergence rate, let us choose $h(\Delta)=\Delta^{-1 / 6}$. Again, let $r=2$ and $p=20$. We already showed in Section 2 that condition $(2.17)$ forces $\Delta \leq 10^{-18}$. In this situation, Theorem 2.5 shows

$$
\mathbb{E}\left|x(T)-x_{\Delta}(T)\right|^{2} \vee \mathbb{E}\left|x(T)-\bar{x}_{\Delta}(T)\right|^{2} \leq C \Delta^{2 / 3}
$$


But, applying Theorem 3.6 we get

$$
\mathbb{E}\left|x(T)-x_{\Delta}(T)\right|^{2} \vee \mathbb{E}\left|x(T)-\bar{x}_{\Delta}(T)\right|^{2} \leq C\left(\Delta^{7 / 9}+\Delta^{2 / 3}\right) \leq C \Delta^{2 / 3}
$$

for any stepsize $\Delta \in(0,1]$. In other words, our new Theorem 3.6 shows the same convergence rate as Theorem 2.5 did without the unrealistic condition $\Delta \leq 10^{-18}$.

Let us discuss one 2-dimensional example to illustrate our theory further.

Example 3.10 Consider a 2-dimensional stochastic Lotka-Volterra system described by

$$
d x(t)=\operatorname{diag}\left(x_{1}(t), x_{2}(t)\right)\left[b+A x^{2}(t)\right] d t+\sigma x(t) d B(t),
$$

where $x^{s}(t)=\left(x_{1}^{s}(t), x_{2}^{s}(t)\right)^{T}$ for any $s \geq 1, b=\left(b_{1}, b_{2}\right)^{T}, \sigma \in \mathbb{R}^{2 \times 2}$,

$$
A=\left(\begin{array}{ll}
-1 & -1 \\
-1 & -1
\end{array}\right) \text {. }
$$

We naturally decompose the coefficients into $f(x)=F_{1}(x)+F(x)$ and $g(x)=G_{1}(x)+G(x)$ with

$$
F_{1}(x)=\left(b_{1} x_{1}, b_{2} x_{2}\right)^{T}, F(x)=\left(\varphi_{1}(x), \varphi_{2}(x)\right)^{T}, G_{1}(x)=\sigma x, G(x)=0,
$$

where

$$
\varphi_{i}(x)=\sum_{j=1}^{2} a_{i j} x_{i} x_{j}^{2}, \quad 1 \leq i \leq 2 .
$$

As both $F_{1}(x)$ and $G_{1}(x)$ are linear functions of $x$, they of course satisfy condition (2.3). For $x, y \in \mathbb{R}^{2}$, noting

$$
\varphi_{i}(x)-\varphi_{i}(y)=\sum_{j=1}^{2} a_{i j}\left(x_{i} x_{j}^{2}-y_{i} y_{j}^{2}\right)=\sum_{j=1}^{2} a_{i j}\left[x_{i}\left(x_{j}^{2}-y_{j}^{2}\right)+\left(x_{i}-y_{i}\right) y_{j}^{2}\right],
$$

we see that there is a positive constant $L_{1}$ such that

$$
|F(x)-F(y)| \leq L_{1}\left(1+|x|^{2}+|y|^{2}\right)|x-y| .
$$

In other words, we have verified Assumption 2.1 as $G(x)=0$. To verify Assumption 2.2, we 
derive that

$$
\begin{aligned}
& (x-y)^{T}(F(x)-F(y)) \\
= & -(x-y)^{T}\left(x|x|^{2}-y|y|^{2}\right) \\
= & -(x-y)^{T}\left((x-y)|x|^{2}+y|x|^{2}-x|y|^{2}+(x-y)|y|^{2}\right) \\
= & -\left(|x-y|^{2}-x^{T} y\right)\left(|x|^{2}+|y|^{2}\right)+2|x|^{2}|y|^{2} \\
= & -\frac{1}{2}|x-y|^{2}\left(|x|^{2}+|y|^{2}\right)-\frac{1}{2}\left(|x|^{2}+|y|^{2}\right)^{2}+2|x|^{2}|y|^{2} \\
= & -\frac{1}{2}|x-y|^{2}\left(|x|^{2}+|y|^{2}\right)-\frac{1}{2}\left(|x|^{2}-|y|^{2}\right)^{2} \\
\leq & 0 .
\end{aligned}
$$

This means that Assumption 2.2 holds for any $\bar{r}>2$. Meanwhile, for any $\bar{p}>2$,

$$
x F(x)+\frac{\bar{p}-1}{2}|G(x)|^{2}=\left(x^{2}\right)^{T} A x^{2} \leq 0 .
$$

Thus, Assumption 2.3 is satisfied. Noting that

$$
\sup _{|x| \leq u}(|F(x)| \vee|G(x)|) \leq\left(|A|_{F} \vee \sigma\right) u^{3}, \quad \forall u \geq 1
$$

which leads $\mu(u)=(3 \vee \sigma) u^{3}$. By using the same method in Example 3.8, we conclude that the order of $L^{r}$-convergence can be arbitrarily close to $1 / 2$ for any $r \geq 2$.

\section{$4 \quad$ Numerical Example}

The convergence rate of the partially truncated EM method was illustrated in [3] by the following example.

Example 4.1 Consider a nonlinear SDE described by

$$
d x(t)=\left(x(t)-x^{5}(t)\right) d t+x^{2}(t) d B(t), t \geq 0,
$$

with the initial value $x(0)=1$.

To our best knowledge, the fully tamed EM method (see [4]) defined by

$$
X_{i+1}=X_{i}+\frac{f\left(X_{i}\right) \Delta+g\left(X_{i}\right) \Delta B_{i}}{\max \left\{1, \Delta\left|f\left(X_{i}\right) \Delta+g\left(X_{i}\right) \Delta B_{i}\right|\right\}},
$$

is also developed for SDEs with both drift and diffusion coefficients growing super-linearly. Consider that the convergence rate of the fully tamed EM method is not obtained in [4], we 


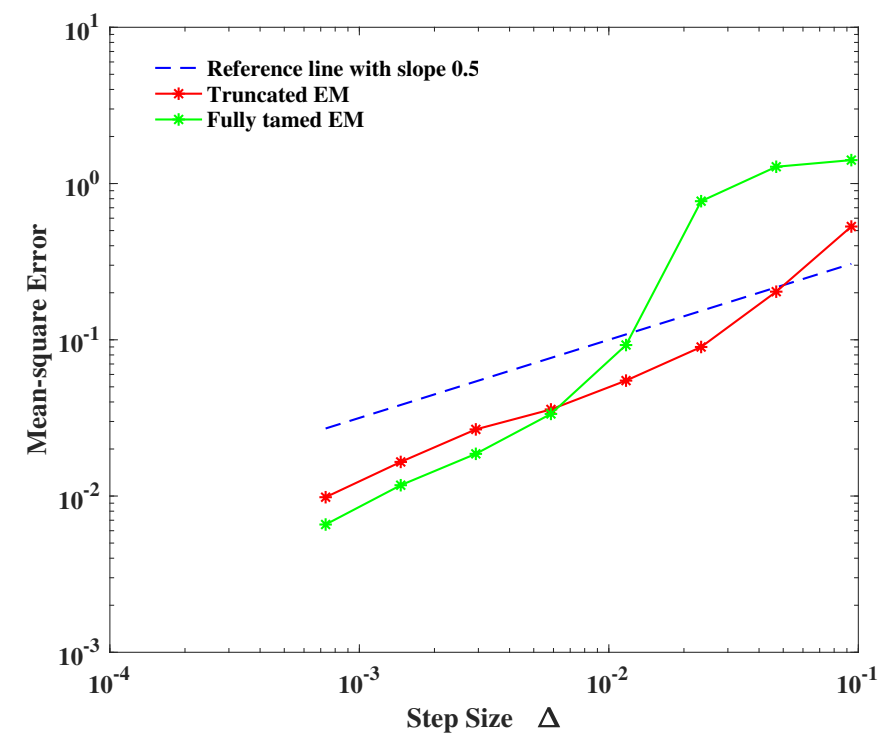

Figure 1: The strong convergence rate at the terminal time $T=3$.

simulate 10,000 sample paths to test the mean-square error. We regard the numerical solutions generated by the partially truncated EM method and the fully tamed EM method in interval $[0,3]$ with the very small step size $\Delta=2^{-12}$ as the 'exact solutions', respectively. Figure 1 displays the $L^{2}$ errors of the fully tamed EM method and the partially truncated EM method at the terminal time $T=3$. It is shown from the figure that both the two methods have the convergence rate of approximately a half for the sufficiently small stepsizes.

Furthermore, we explore possibility of preserving positivity of the two methods using the simulation results. The true solution of the SDE (4.1) starting from a positive initial value will never cross the origin, see Appendix for the proof. It can be seen from the 3000 simulated paths with the stepsize $\Delta=2^{-7}$ in Figures 2 and 3 that the partially truncated EM method has higher chance to preserve the positivity than the fully tamed method. It should be noted that the structure of either the partially truncated EM or the fully tamed method would not guarantee non-negative numerical solutions. Therefore, it would be interesting to conduct some rigorous analyses on the probability of non-negative solutions of both the two methods. We have been working on this topic and will report it in the future work.

\section{Conclusion}

The main contribution of this paper is that we removed a restrictive condition imposed in [3] but still are able to establish the finite-time strong $L^{r}$-convergence for highly nonlinear SDEs. 


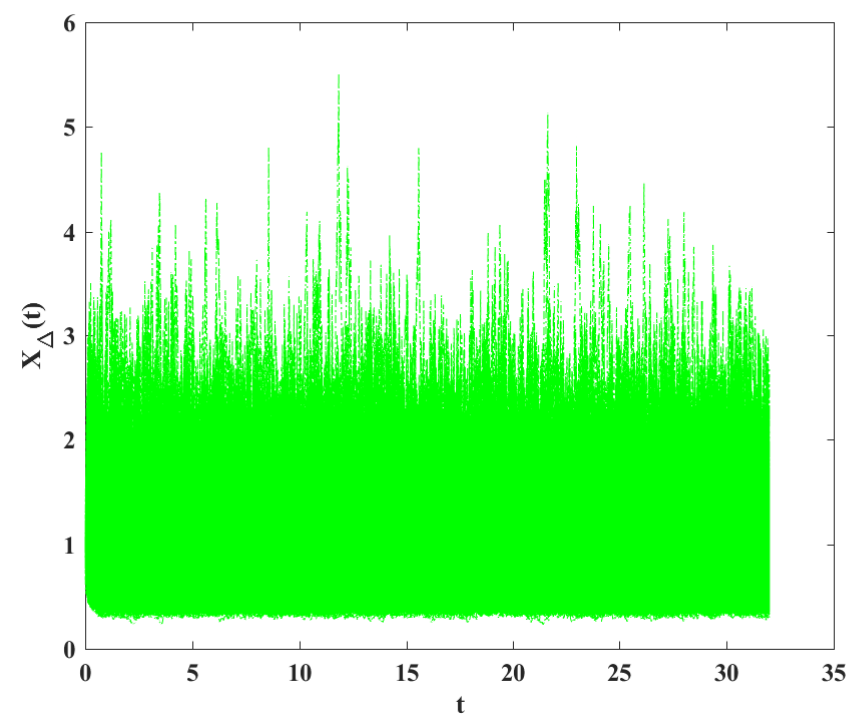

Figure 2: The 3000 sample paths generated by the partially truncated EM method.

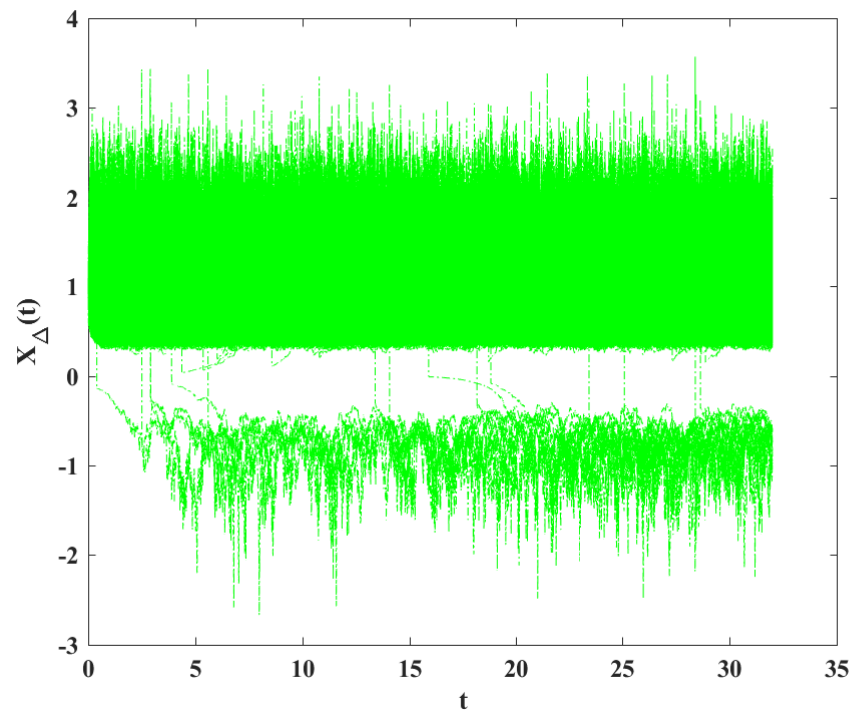

Figure 3: The 3000 sample paths generated by the fully tamed EM method. 
A couple of examples were used to highlight the advantages of our new results compared with the earlier ones in [3]. New techniques were developed in this paper to obtain the results.

\section{Appendix}

Now we prove almost all the sample path of $d x(t)=\left(x(t)-x^{5}(t)\right) d t+x^{2}(t) d B(t)$ starting from a non-zero state, given $x(0)>0$, will never reach the origin.

Define the stopping time,

$$
\tau_{k}=\inf \left\{t>0: x(t) \notin\left[\frac{1}{k}, k\right]\right\}
$$

Clearly, $\tau_{k}$ is increasing as $k \rightarrow \infty$. Set $\tau_{\infty}=\lim _{n \rightarrow \infty} \tau_{k}$, if we can prove $\tau_{k} \stackrel{a . s}{\rightarrow} \infty$ as $k \rightarrow \infty$, then $x(t)>0$ a.s. for all $t>0$. That is to say, to complete the proof, we need to show that $\tau_{\infty}=\infty$. To prove this, for any constant $T$, if $P\left\{\tau_{k} \leq T\right\} \rightarrow 0$ as $k \rightarrow \infty$, then we have $P\left\{\tau_{\infty}=\infty\right\}=1$, which is the required assertion. For $\theta \in(0,1)$, define a $C^{2}$ - function $V:(0, \infty) \rightarrow(0, \infty)$ by

$$
V(x)=x^{\theta}-1-\theta \log (x)
$$

It is clear that $V(\cdot) \geq 0$ and $V(x) \rightarrow \infty$ as $x \rightarrow \infty$ or $x \rightarrow 0$. Applying the Itô formula yields

$$
\begin{gathered}
d V(x(t))=\left[\left(\theta x^{\theta-1}(t)-\frac{\theta}{x(t)}\right)\left(x(t)-x^{5}(t)\right)+\frac{1}{2}\left(\theta(\theta-1) x^{\theta-2}(t)+\frac{\theta}{x^{2}(t)}\right) x^{4}(t)\right] d t \\
+\left(\theta x^{\theta-1}(t)-\frac{\theta}{x(t)}\right) x^{2}(t) d B(t)
\end{gathered}
$$

By boundedness of polynomial, for $\theta \in(0,1)$, there exists a constant $K_{1}$ such that

$$
\theta x^{\theta}-\theta x^{\theta+4}-\theta+\theta x^{4}+\frac{1}{2} \theta(\theta-1) x^{\theta+2}+\frac{1}{2} \theta x^{2} \leq K_{1} .
$$

Therefore, for any $t \in[0, T]$,

$$
\mathbb{E} V\left(x\left(t \wedge \tau_{k}\right)\right) \leq V(x(0))+K_{1} T
$$

So

$$
P\left\{\tau_{k} \leq T\right\}\left[V\left(\frac{1}{k}\right) \wedge V(k)\right] \leq \mathbb{E} V\left(x\left(T \wedge \tau_{k}\right)\right) \leq V(x(0))+K_{1} T .
$$

Then we have $P\left\{\tau_{k} \leq T\right\} \rightarrow 0$ since $V\left(\frac{1}{R}\right) \wedge V(k) \rightarrow \infty$ as $k \rightarrow \infty$.

That is

$$
P\left\{\tau_{\infty}=\infty\right\}=1
$$




\section{Acknowledgements}

The authors would like to thank the Natural Science Foundation of Shanghai (14ZR1431300), Shanghai Pujiang Program (16PJ1408000), the National Natural Science Foundation of China

(11701378), "Chenguang Program" supported by Shanghai Education Development Foundation and Shanghai Municipal Education Commission (16CG50), the Natural Science Fund of Shanghai Normal University (SK201603), the EPSRC (EP/K503174/1), the Leverhulme Trust (RF-2015-385), the Royal Society (Wolfson Research Merit Award WM160014), the Royal Society and the Newton Fund (NA160317, Royal Society-Newton Advanced Fellowship) and the Ministry of Education (MOE) of China (MS2014DHDX020), for their financial support.

\section{References}

[1] Bahar, A. and Mao, X., Stochastic delay population dynamics, J. Int. Appl. Math. $11(4)(2004), 377-400$.

[2] Ginzburg, V. L. and Landau, L. D., On the theory of superconductivity, Zh. Eksperim. i teor. Fiz. 20(1950), 1064-1082.

[3] Guo, Q., Liu, W., Mao, X. and Yue, R., The partially truncated Euler-Maruyama method and its stability and boundedness, Appl. Numer. Math. 115 (2017), 235-251.

[4] Hutzenthaler, M., Jentzen, A., Numerical approximations of stochastic differential equations with non-globally Lipschitz continuous coefficients, Mem. Amer. Math. Soc. 236(2) (2015) 99 pages.

[5] Khasminskii R.Z. , Stochastic Stability of Differential Equations, Alphen: Sijtjoff and Noordhoff, 1980. (Translation of the Russian edition, Moscow, Nauka 1969).

[6] Kloeden, P. E. and Platen, E., Numerical Solution of Stochastic Differential Equations, Springer-Verlog, Berlin, 1992.

[7] Mao, X., Stochastic Differential Equations and Applications, 2nd Edition, Horwood, Chichester, UK, 2007.

[8] Mao, X., The truncated Euler-Maruyama method for stochastic differential equations, J. Comput. Appl. Math. 290 (2015), 370-384. 
[9] Mao, X., Convergence rates of the truncated Euler-Maruyama method for stochastic differential equations, J. Comput. Appl. Math. 296 (2016), 362-375.

[10] Mao, X. and Yuan, C., Stochastic Differential Equations with Markovian Switching, Imperial College Press, 2006.

[11] Song, M., Hu, L., Mao, X. and Zhang, L., Khasminskii-Type theorems for stochastic functional differential equations, Discrete Contin. Dyn. Syst. Ser. B 18(6) (2013), 16971714. 